\title{
Steady-State Kalman Estimator for Descriptor Systems with Colored Noise
}

\author{
Yan $\mathrm{Xu}$ and Guosheng Zhang \\ Beijing Institute of Graphic Communication, Beijing 102600, China
}

\begin{abstract}
Using the modern time series analysis method in the time domain, based on the ARMAinnovation model, a steadystate Kalman estimator for descriptor systems with colored noise is introduced,and employing the state observer principle, the pole-assignment descriptor steady-state Kalman estimator is also presented. They have global asymptotic stability and can handle the filtering, smoothing and prediction problems in unified frameworks, thus avoiding the solution of the Riccatiequations.
\end{abstract}

Keywords-escriptor systems; colored noise; steady-state Kalman estimator; global asymptoticstability

\section{INTRODUCTION}

Descriptor systems often occur in many fields including circuit, economics, robotics, etc, and have attracted considerable attention in recent years[1]. Steady-stateKalman estimators fordescriptor systems in [2] and [3]are limited to white noises, and the noises of the practical problems are often not the ideal white noises. The descriptor steady-state Kalman estimator of [2] have a shortcoming: it may be nonasymptotically stableand the optimal initial valueis required. In this paper, a steady-state Kalman estimator for descriptor systems with colored noise is given. It overcomes the limitations of white noise. In order to obtain the global asymptotic stability of the estimator, the pole-assignment descriptor steady-state Kalman estimator is presented by using the principle of the state observer. Not only it has the global asymptotic stability, but also the effect of theinitial state estimate can be rapidly forgotten by assigning the pole of the estimator.

Consider the discrete time descriptor stochastic system

$$
\begin{gathered}
M x(t+1)=\Phi x(t)+\Gamma w(t), \\
y(t)=H x(t)+v(t),
\end{gathered}
$$

where the state $x(t) \in R^{n}$, the measurement $y(t) \in R^{m}$, $w(t) \in R^{r}, v(t) \in R^{m}, M, \Phi, \Gamma$ and $H$ are the constant matrices.

\section{Assumption 1}

The $n \times n$ matrix $M$ is singular, that is

\section{Assumption 2}

$$
\operatorname{det}(M)=0
$$

The system is completely observable, that is

$$
\begin{aligned}
& \operatorname{rank}\left[\begin{array}{c}
z M-\Phi \\
H
\end{array}\right]=n, \\
& \forall z \in C ; \quad \operatorname{rank}\left[\begin{array}{c}
M \\
H
\end{array}\right]=n .
\end{aligned}
$$

\section{Assumption 3}

The system is regular, that is

$$
\forall z \in C, \operatorname{det}(z M-\Phi) \not \equiv 0 .
$$

Assumption 4

$v(t)$ is the white noise with zero mean, and $w(t)$ is the zero meancolored noise correlated with $v(t)$, and

$$
\begin{gathered}
\mathrm{E}\left[w(t) w^{\mathrm{T}}(t)\right]=W_{0}, \\
\mathrm{E}\left[w(t) w^{\mathrm{T}}(t+1)\right]=W_{1}, \\
\mathrm{E}\left[w(t) w^{\mathrm{T}}(t+j)\right]=0(\mathrm{j} \geq 2), \\
\mathrm{E}\left[w(t) v^{\mathrm{T}}(t)\right]=S_{0}, \\
\left.\mathrm{E}\left[v(t) v^{\mathrm{T}}(j)\right]=V(t) v^{\mathrm{T}}(t+1)\right]=S_{1}, \\
\mathrm{E}\left[w(t) v^{\mathrm{T}}(t+j)\right]=0(\mathrm{j} \geq 2), \\
\mathrm{E}\left[w(t) v^{\mathrm{T}}(t-j)\right]=0(\mathrm{j} \geq 1) .
\end{gathered}
$$

\section{ARMA INNOVATION MODEL}

From (1)and (2)we have

$$
y(t)=H\left(M-q^{-1} \Phi\right)^{-1} \Gamma q^{-1} w(t)+v(t),
$$

where $q^{-1}$ is the backward shift operator. Introducing the leftcoprimefactorization

$$
H\left(M-q^{-1} \Phi\right)^{-1} \Gamma q^{-1}=A^{-1} B q^{\tau},
$$


where $A$ and $B$ are polynomial matrices having the form

$$
\begin{gathered}
X=X\left(q^{-1}\right)=X_{0}+X_{1} q^{-1}+\Lambda+X_{n_{x}} q^{-n_{x}}, \\
A_{0}=I_{m}, B_{0} \neq 0,
\end{gathered}
$$

$\tau$ is an integer.

Substituting (6)into (5)yields the ARMA innovation model

$$
\begin{gathered}
A y(t)=D e(t), \\
D e(t)=B q^{\tau} w(t)+A v(t),
\end{gathered}
$$

where $D$ is a stable polynomial matrix, $D_{0}=I_{m} \cdot e(t) \in R^{m}$ is thewhite noise with zero mean and variance matrix $Q_{e} . D$ and $Q_{e}$ can be obtained by using the Gevers-Wouters algorithm[4]. According to (7), the innovations $e(t)$ can be computed recursively as

$$
\begin{gathered}
e(t)=A y(t)-D_{1} e(t-1)-\Lambda-D_{n_{d}} e\left(t-n_{d}\right), \\
t=n_{d}, n_{d}+1 \Lambda .
\end{gathered}
$$

with the initial values $\left(e(0), \Lambda, e\left(n_{d}-1\right)\right)$.And the spectral matrix of the random process in (8)is assumed nonsingular[5].

\section{NOISE ESTIMATORS}

Theorem 1

Under Assumptions 1 4, the system (1) (2) have

$$
\begin{gathered}
\mathrm{E}\left[w(t) e^{\mathrm{T}}(j)\right]=J_{j-t}, \\
\mathrm{E}\left[v(t) e^{\mathrm{T}}(j)\right]=L_{j-t},
\end{gathered}
$$

then we have the colored noiseestimators

$$
\begin{aligned}
& \hat{w}(t \mid t+N)=\sum_{i=-(\tau \vee 0)-1}^{N} J_{i} Q_{e}^{-1} e(t+i) \\
& \hat{v}(t \mid t+N)=\sum_{i=-(\tau \vee 0)-1}^{N} L_{i} Q_{e}^{-1} e(t+i)
\end{aligned}
$$

where

$$
\begin{aligned}
J_{i}= & W_{0} F_{i+(\tau \vee 0)}^{\mathrm{T}}+W_{1} F_{i+(\tau \vee 0)-1}^{\mathrm{T}}+W_{1}^{\mathrm{T}} F_{i+(\tau \vee 0)+1}^{\mathrm{T}} \\
& +S_{0} G_{i+(\tau \vee 0)}^{\mathrm{T}}+S_{1} F_{i+(\tau \vee 0)-1}^{\mathrm{T}}(t \geq 1) \\
& L_{i}=S_{0}^{\mathrm{T}} F_{i+(\tau \vee 0)}^{\mathrm{T}}+S_{1}^{\mathrm{T}} F_{i+(\tau \vee 0)+1}^{\mathrm{T}}+V G_{i+(\tau \vee 0)}^{\mathrm{T}},
\end{aligned}
$$

where

$$
\begin{aligned}
& (a \vee b)=\max (a, b), \\
& (a \wedge b)=\min (a, b),
\end{aligned}
$$

and $F_{i}, G_{i}$ can be computed recursively as

$$
\begin{gathered}
F_{i}=-D_{1} F_{i-1}-\Lambda \Lambda-D_{n_{d}} F_{i-n_{d}}+\bar{B}_{i}, \\
F_{i}=0(i<0), \bar{B}_{i}=0\left(i>n_{\bar{b}}\right) \\
G_{i}=-D_{1} G_{i-1}-\Lambda \Lambda-D_{n_{d}} G_{i-n_{d}}+\bar{A}_{i}, \\
G_{i}=0(i<0), \bar{A}_{i}=0\left(i>n_{\bar{a}}\right),
\end{gathered}
$$

And

Proof.

$$
\begin{aligned}
& \bar{B}\left(q^{-1}\right)=B\left(q^{-1}\right) q^{(\tau \wedge 0)}, \\
& \bar{A}\left(q^{-1}\right)=A\left(q^{-1}\right) q^{(-\tau \wedge 0)} .
\end{aligned}
$$

According to the proof method of [4] under Assumption 4, we get

$$
\begin{aligned}
& \mathrm{E}\left[u(t) e^{\mathrm{T}}(j)\right]=J_{j-t}, \\
& \mathrm{E}\left[v(t) e^{\mathrm{T}}(j)\right]=L_{j-t},
\end{aligned}
$$

Then from the projection property, we have

$$
\begin{aligned}
& \hat{w}(t \mid t+N)=\sum_{j=0}^{t+N} \mathrm{E}\left[w(t) e^{\mathrm{T}}(j)\right] Q_{e}^{-1} e(j), \\
& \hat{v}(t \mid t+N)=\sum_{j=0}^{t+N} \mathrm{E}\left[v(t) e^{\mathrm{T}}(j)\right] Q_{e}^{-1} e(j) .
\end{aligned}
$$

Then thecolored noiseestimators (11) (13) can be obtained.

$$
\text { IV. LEMMAS[4] }
$$

\section{Lemma 1}

$$
\mathrm{E}\left[y(t) e^{\mathrm{T}}(j)\right]=P_{t-j} Q_{e}
$$

where

$$
\begin{gathered}
P_{i}=-A_{1} P_{i-1}-\Lambda \Lambda-A_{n_{a}} P_{i-n_{a}}+D_{i}, \\
P_{i}=0(i<0), D_{i}=0\left(i>n_{d}\right) .
\end{gathered}
$$

From Assumptions 2 we know

$$
\operatorname{rank}\left[M^{\mathrm{T}} \quad H^{\mathrm{T}}\right]^{\mathrm{T}}=n .
$$

Then there exists an $n \times m$ matrix $T_{0}$ such that $\left(M+T_{0} H\right)$ is nonsingular. Premultiplying (2) by $T_{0}$, and combining it with (1) yield

$$
x(t)=\Psi x(t-1)+\Psi_{1} y(t)-\Psi_{1} v(t)+\Psi_{2} w(t-1)
$$

where 


\section{Lemma 2}

$$
\begin{aligned}
& \Psi=\left(M+T_{0} H\right)^{-1} \Phi, \\
& \Psi_{1}=\left(M+T_{0} H\right)^{-1} T_{0}, \\
& \Psi_{2}=\left(M+T_{0} H\right)^{-1} \Gamma .
\end{aligned}
$$

System (1) (2) have the completely observable pair $(\Psi, H)$ under Assumptions 1 4.

\section{Lemma 3}

System (1) (2) have the non-recursive state expression under Assumptions 1 4 as

$$
\begin{gathered}
x(t)=\sum_{i=0}^{\beta-1} \Omega_{i}\left[y(t+i)-\sum_{j=0}^{i-1} H \Psi^{i-1-j}\left[\Psi_{1} y(t+j+1)\right.\right. \\
\left.\left.+\Psi_{1} v(t+j+1)+\Psi_{2} w(t+j)\right]-v(t+i)\right],
\end{gathered}
$$

where

$$
\begin{gathered}
\Omega=\left[\begin{array}{c}
H \\
H \Psi \\
\mathrm{M} \\
H \Psi^{\beta-1}
\end{array}\right], \\
\Omega^{\#}=\left(\Omega^{\mathrm{T}} \Omega\right)^{-1} \Omega^{\mathrm{T}}=\left[\Omega_{0}, \Omega_{1}, \Lambda, \Omega_{\beta-1}\right],
\end{gathered}
$$

and we define

$$
\Psi^{i}=0(i<0), j \geq 0
$$

$\beta$ is the observability index, satisfy

$$
n m^{-1} \leq \beta \leq n-m+1 .
$$

\section{DEscriptorSteady State KaLMAN Estimators}

Theorem 2

Under Assumptions 1 4, the system (1) (2) have the descriptor steady state Kalmanestimator as

$$
\begin{gathered}
\hat{x}(t \mid t+N)=\Psi \hat{x}(t-1 \mid t-1+N)+\Psi_{1} \hat{y}(t \mid t-1+N) \\
-\Psi_{1} \hat{v}(t \mid t-1+N)+\Psi_{2} \hat{w}(t-1 \mid t-1+N) \\
+K_{N} e(t+N),
\end{gathered}
$$

where

$$
\begin{aligned}
& K_{N}=\sum_{i=0}^{\beta-1} \Omega_{i}\left\{P_{i-N}-\sum_{j=0}^{i-1} H \Psi^{i-1-j}\left[\Psi_{1} P_{j+1-N}\right.\right. \\
& \left.\left.-\Psi_{1} L_{N-j-1} Q_{e}^{-1}+\Psi_{1} J_{N-j} Q_{e}^{-1}\right]-L_{N-i} Q_{e}^{-1}\right\}
\end{aligned}
$$

and in (18), we define

$$
\Psi^{i}=0(i<0), j \geq 0 .
$$

$\hat{w}(i \mid j)$ and $\hat{v}(i \mid j)$ can be computed by Theorem $1 . \hat{y}(i \mid j)$ can be computed recursivelyas

$$
\hat{y}(i \mid j)=-A_{1} \hat{y}(i-1 \mid j)-\Lambda \Lambda-A_{n_{a}} \hat{y}\left(i-n_{a} \mid j\right)
$$

$$
+D e(i), \quad(i>j)
$$

where

and

$$
e(k)=0,(k>j),
$$

Proof.

$$
\hat{y}(i \mid j)=y(i),(i \leq j) .
$$

According to the projection property, we have

$$
\hat{x}(t \mid t+N)=\hat{x}(t \mid t-1+N)+K_{N} e(t+N),
$$

$$
K_{N}=\mathrm{E}\left[x(t) e^{\mathrm{T}}(t+N)\right] Q_{e}^{-1} .
$$

Taking the projection operation for (15) yields

$$
\begin{gathered}
\hat{x}(t \mid t-1+N)=\Psi \hat{x}(t-1 \mid t-1+N)+\Psi_{1} \hat{y}(t \mid t-1+N) \\
-\Psi_{1} \hat{v}(t \mid t-1+N)+\Psi_{2} \hat{w}(t-1 \mid t-1+N)
\end{gathered}
$$

Substituting (21) into (20) yields (17), Substituting (16), (10) and (14)into (20) yields (18).

Theorem 3

If $\Psi$ is a stable matrix, then the descriptor steady-state Kalman estimators (17) are globally asymptotically stable, that is, $\hat{x}(t \mid t+N)$ are asymptotically independent ofboth the initial estimates $\hat{x}\left(t_{0} \mid t_{0}+N\right)$ and the innovation initial values $\left(e(0), \Lambda, e\left(n_{d}-1\right)\right)$.

Proof.

The proof is similar to that as in [4] , which is omitted.

Theorem 4

If $\Psi$ is an unstable matrix, then for the system (1) and (2) under Assumptions 1 4, we can suitably select an $n \times m$ matrix $\bar{T}_{0}$, such that $\bar{\Psi}=\Psi+\bar{T}_{0} H$ is stable, so that the pole assignment descriptor steady-state Kalman estimators have globally asymptotic stability as

$$
\begin{gathered}
\hat{x}(t \mid t+N)=\bar{\Psi} \hat{x}(t-1 \mid t-1+N)+\Psi_{1} \hat{y}(t \mid t-1+N) \\
-\bar{T}_{0} \hat{y}(t-1 \mid t-1+N)-\Psi_{1} \hat{v}(t \mid t-1+N) \\
+\bar{T}_{0} \hat{v}(t-1 \mid t-1+N)+\Psi_{2} \hat{w}(t-1 \mid t-1+N)
\end{gathered}
$$




$$
+K_{N} e(t+N)
$$

Proof.

The proof is similar to that as in [4], which is omitted.

Note.

According to the principle of state observer[6], in order to ensure that the effect of the initial values $\hat{x}\left(t_{0} \mid t_{0}+N\right)$ is rapidlyforgotten, we usually assign the eigenvalues of $\bar{\Psi}$ values close to the origin. If we assign theeigenvalues of $\bar{\Psi}$ values close to the bound of the unit circle, then although we can make $\bar{\Psi}$ astable matrix, but the effect of the initial values $\hat{x}\left(t_{0} \mid t_{0}+N\right)$ will be forgotten in a longer decayingprocess.

Secondly, theorem 4 includes Theorem 3 as a special case. When $\Psi$ is stable, we select $\bar{T}_{0}=0$. Theorem 4 can also be used to the case that $\Psi$ is a stable matrix and its eigenvalues are approximate to the unitcircle. We can assign new eigenvalues of $\Psi$ in order that the effect ofthe initial values $\hat{x}\left(t_{0} \mid t_{0}+N\right)$ can be rapidly forgotten.

\section{CONCLUSION}

Using the modern time series analysis method, the steadystate Kalman estimator for descriptor system with colored noise is presented. It overcomes the limitations of previous estimators for only applying to white noise, and avoids the solution of the Riccatiequations. The algorithm is simple and easy to use. In order to obtain the global asymptotic stability of the estimator, the pole-assignment descriptor steady-state Kalman estimator with the global asymptotic stability is also presented by using the principle of the state observer. Compared with [1] and [2], it avoids computingthe optimal initial value of stateestimator. And the computational burden is reduced.Itismore suitable for real time applications.

\section{ACKNOWLEDGMENTS}

The research work was supported by the Foundation of Beijing Municipal Education Commissionunder Grant No.KM2014100150012, Projects on (Building) ExcellentTeaching Team of Beijing Institute of GraphicCommunication (22150114004) and Beijing high school youth talent plan (YETP1471).

\section{REFERENCES}

[1] R.Nikoukhah, A.S.Willsky, and C.L.Bernard, "Kalman filtering and Riccatiequations for descriptor systems,” IEEE Transactions on Automati Control, vol.37(9), pp.1325-1341, 1992.

[2] Z. L. Deng and Y. Xu, "New approaches to Wiener filtering and Kalman filtering for descriptor systems,"Chinese Journal of Control Theory and Applications, vol 16(5), pp. 634-638, 1999.

[3] Z. L. Deng and Y.M. Liu, “Descriptor Kalman estimators,” International Journal of Systems Science, vol 30(11), pp. 1205-1212, 1999.

[4] Z. L. Deng, "Optimal Filtering Theory and Applications: Modern Time Series Analysis Method”,Harbin :Harbin Institute of Technology Press, 2000.

[5] J. G. Chen, Z. Q. Shi, and P. D. Wang, "A Generalization of Kalman Filtering Theory," Chinese Journal of Control Theory and Applications, vol7(3), pp.108-112, 1990.
[6] C. T. Chen, "Linear System Theory and Design," New York: Holt, Rinehart and Winston, 1984.

[7] Z. L. Deng, H. S. Zhang,S. J. Liu, and L. Zhou, "Optimal and self-tuning white noise estimators with applications to deconvolution and filteringproblems,” Automatica, vol 32(2), pp. 199-216, 1996.

[8] Z. L. Deng and Y. X.Guo, "Modern Time Series Analysis and Its Applications:Modelling, Filtering, Deconvolution, Predicition and Control,” Beijing: Knowledge Press, 1989.

[9] Z. L. Deng and Y.M. Liu, “Steady-state Kalman estimators for singular systems," Chinese Journal of Acta Automatica Sinica, vol25(4), pp.483487, 1999. 